\title{
Electrochemical Self-Assembly of Nanoporous Alumina Templates Title
}

\author{
Muna Moussa Abbas \\ Department of Physics, College of Science, Baghdad University, Baghdad, Iraq \\ Email:muna_moussa@yahoo.com
}

Received May 21, 2013; revised June 25, 2013; accepted July 12, 2013

Copyright (C) 2013 Muna Moussa Abbas. This is an open access article distributed under the Creative Commons Attribution License, which permits unrestricted use, distribution, and reproduction in any medium, provided the original work is properly cited.

\begin{abstract}
Porous alumina was fabricated electrochemically through anodic oxidation of aluminum by means of such a self-organized method. Anodic aluminium oxide (AAO) template with nanopores was grown by two-steps anodization processes from a high purity aluminium foil. The anodization process was carried out in a phosphoric acid electrolyte at ambient temperature with a different duration of anodization. The analysis observation by Atomic Force Microscopy (AFM) showed that nanopore size increased with anodization time. The nanopore sizes of porous alumina were (16.04, 26.19 and $37.39 \mathrm{~nm}$ ) for (1, 2 and 3 hour) respectively.
\end{abstract}

Keywords: Anodic Alumina; Porous Alumina; Self-Assembly

\section{Introduction}

The discovery of nanoporous materials opened a tremendous flurry of worldwide research, because of their promising and potential industrial and technological applications for magnetic, electronic, optoelectronic structures and nanometric devices.

Moreover, the application of ordered nanochannel-arrays as two-dimensional photonic crystals has generated an increasing interest in recent years. Anodic porous alumina film is a promising candidate for starting materials of nanofabrication of various devices [1,2].

Porous alumina is arrays of a large $2 \mathrm{D}$ adjustable configuration forms on the surface of aluminum in a selforganized manner when anodized in an acid under specific conditions $[3,4]$.

Since Masuda et al. [5] reported the two-steps anodization process to obtain a self-ordered of pores, new areas of applications have emerged in the fields of magnetic storage, solar cells, carbon nanotubes, catalysts, and metal nanowires. This increasing attraction of porous alumina as template is mainly due to it enables mass production without the use of expensive lithographical tools [6].

Anodic aluminium oxide (AAO) templates have received considerable attention in synthesizing nanostructure material due to their particular characteristics such as controllable pore diameter and periodicity, and extremely narrow distribution of pore size. The pore has ideal hexagonal shape. AAO is characterized by a homogeneous morphology of parallel pores which grow perpendicular to the template surface with a narrow distribution of diameter, length and interpore spacing, all of which can be easily controlled by a suitable way of choosing the anodization parameters such as $\mathrm{pH}$ of the electrolyte, anodization voltage and duration of anodization $[7,8]$.

Wang and Han [9] showed that AAO preparation after a two-steps anodization possesses controllable pore diameter and periodicity; therefore further pore adjustment can be caused by exposure to phosphoric acid, which is called pore-widening.

Extensive researches have been carried to a better understanding of the physical and chemical properties of porous alumina. The preparing conditions of highly ordered AAO template are highly precise, especially for those with pore diameters under $25 \mathrm{~nm}$. The anodization time is one of the main factors of fabrication for the order of nanopore arrays.

Nielsch et al. [10] showed that the domain size does not linearly increase with time but has a maximum size and he suggested that it is an optimum anodization time to obtain the largest domain size. This goes beyond the results of Li et al. [11], Oh et al. [12] and Zaraska et al. [13] they reported that the average domain area is a linear function of anodization time.

Lee et al. [14] investigated the anodization of aluminum in malonic acid $\left(\mathrm{H}_{4} \mathrm{C}_{3} \mathrm{O}_{4}\right)$ solution. They found that 
the ordering of cells shows an inverse time dependence on the anodization time.

The purpose of this work is to understand and improve the self-assemble of porous alumina under a specific condition with the variation of duration of the anodization.

\section{Experimental Details}

The AAO templates were fabricated by a two-step anodization process. Specimens of High purity $(99.93 \%, 0.3$ $\mathrm{mm}$ thick) aluminium foils annealed at $500^{\circ} \mathrm{C}$ for 3 hour in an $\mathrm{N}_{2}$ atmosphere then it was purged by acetone in an ultrasonic cleaner to clean out the possible grease on its surface. To reduce surface roughness, electropolishing is carried out in a mixture consisting of 1:4 volume mixture of $\left(\mathrm{HClO}_{4}\right)$ and $\left(\mathrm{C}_{2} \mathrm{H}_{5} \mathrm{OH}\right)$ at $10 \mathrm{~V}$ for 3 min at $10^{\circ} \mathrm{C}$ with magnetic stirring. The samples were later rinsed in distilled water; this step repeats ones again under the same conditions. After the electrochemically polishing procedure, the specimens were anodized in two electrodes electrochemical cell with a magnetic stirrer was used, $\mathrm{Pt}$ foil serves as cathode where the $\mathrm{Al}$ foil as the anode, the electrolyte was $10 \%$ sulfuric acid, the process conducted with $18 \mathrm{~V} \mathrm{DC}$ at $5^{\circ} \mathrm{C}$ for different duration of anodizing (1, 2 and 3 hour). A two steps anodizing procedure were conducted in which the first anodized layer removed by solution of $(1.8 \mathrm{wt}$ chromic acid and $6 \% \mathrm{wt}$ phosphoric acid) at $60^{\circ} \mathrm{C}$. The samples cleaned again as mentioned above to repeat the anodizing process at the same conditions of the first step, then immersed in $6 \%$ of phosphoric acid to open the pores where the phosphoric acid is thought to help connect cylindrical pores in the bottom layer to the top layer of the AAO template.

Atomic Force Microscopy (AFM-AA3000) of Angstrom Advanced Inc., USA) was used to obtain quantitative information on the grain sizes of the AAO films as well as the surface roughness.

\section{Results and Discussion}

The morphology in the three-dimensional AFM images and the statistical results of the anodized aluminum porous films in a three different times, $(1-3$ hour $)$ are shown in Figures (1-3) respectively. The results show distinctly that an ordered honeycomb structure with this method.

It was observed that the pore diameter is $16.04 \mathrm{~nm}$ for AAO prepared under 1 hour, increasing the time up to 2 hour produced AAO with a pore of $26.19 \mathrm{~nm}$ size in diameter as shown in Figure 2. In addition, the pores were possessed irregular structure and separated by a thick pore walls. The pores were continuity formed on the surface; its structure becomes more open by increasing the anodizing time up to 3 hour. The measuring of the pore

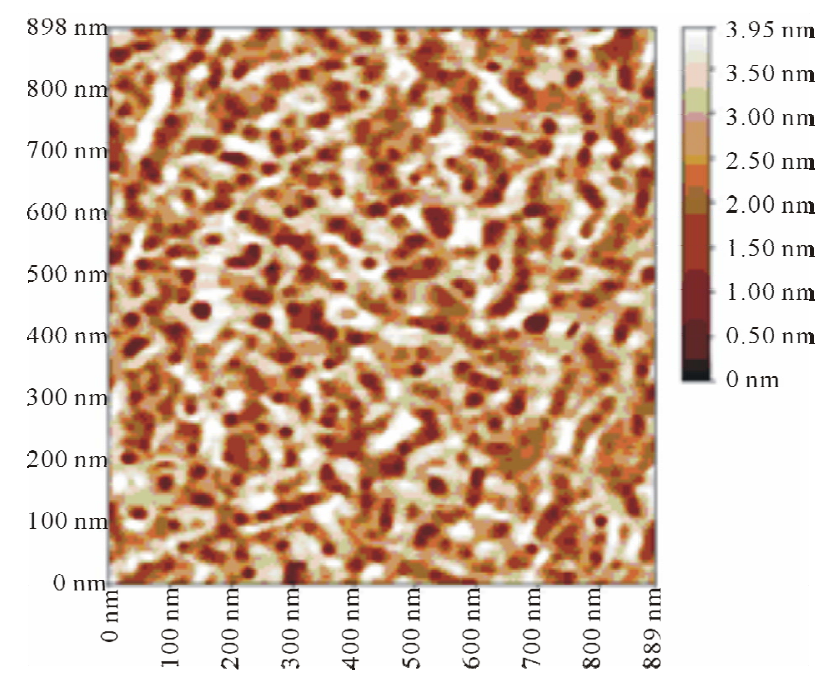

(a)

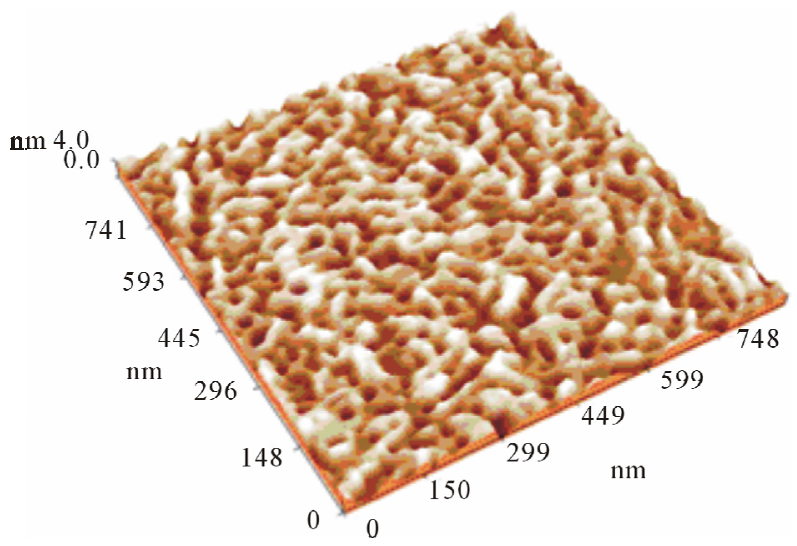

(b)

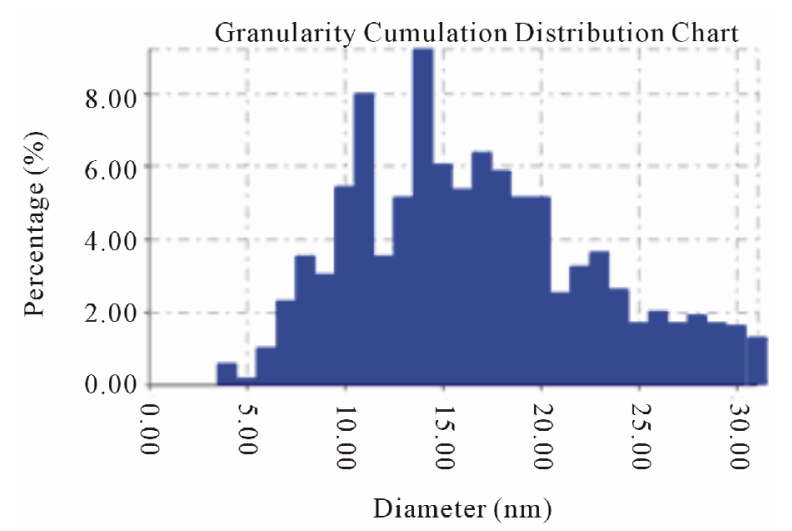

(c)

Figure 1. Atomic force microscope topographic views of AAO ionized during 1 hour (a) 2D image (b) 3D and (c) normal granulites distribution report of AAO.

diameter appeared to be $37.39 \mathrm{~nm}$ as illustrates in Figure 3. The structure of the pore was more circular with a large opening and separated by a thick pore walls.

Meanwhile, the pores were more circular with a large opening and separated by a thick pore walls; it had been 


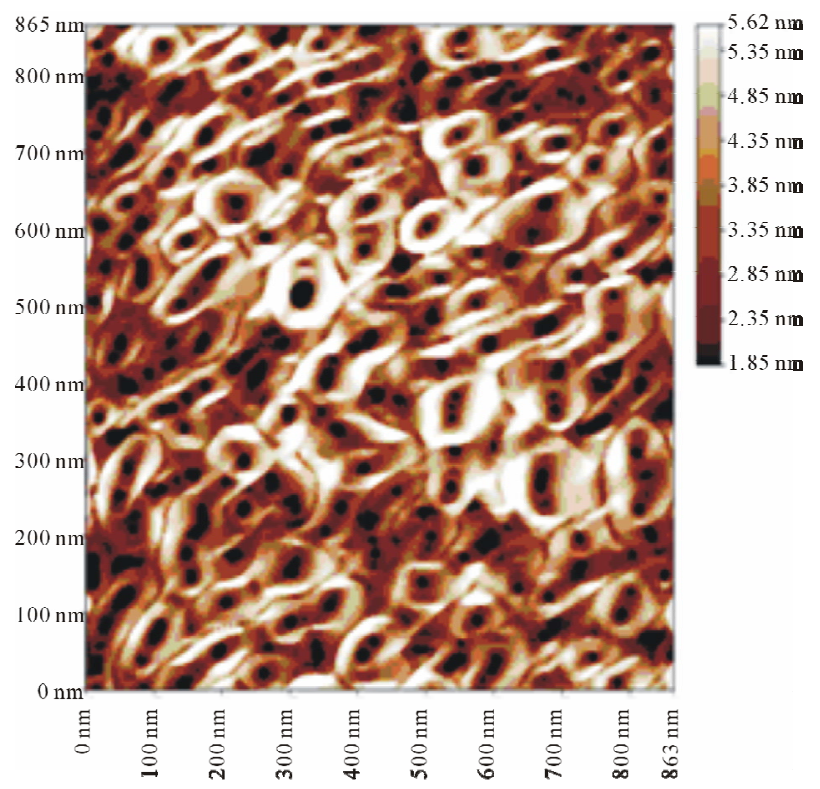

(a)

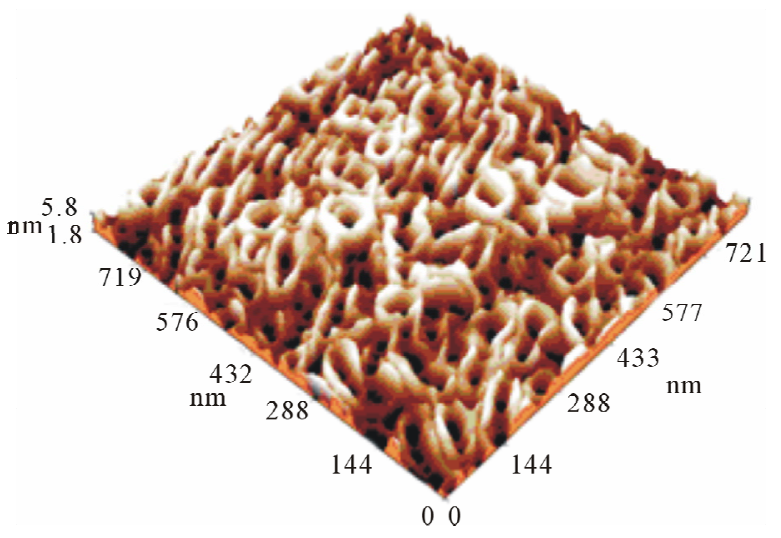

(b)

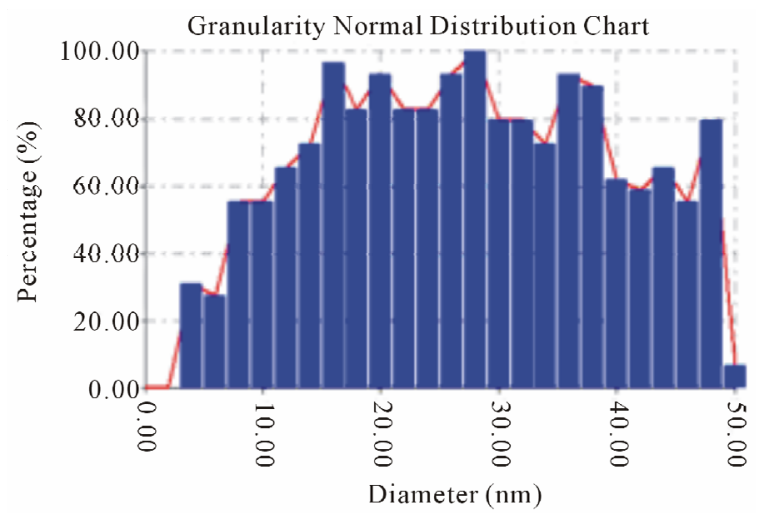

(c)

Figure 2. Atomic force microscope topographic views of AAO ionized during 2 hour (a) 2D image (b) 3D and (c) normal granulites distribution report of AAO.

shown that the size distribution become inhomogeneous at longer anodization time. It can be conclude that connection of pores is an intermediate step in porous alu-

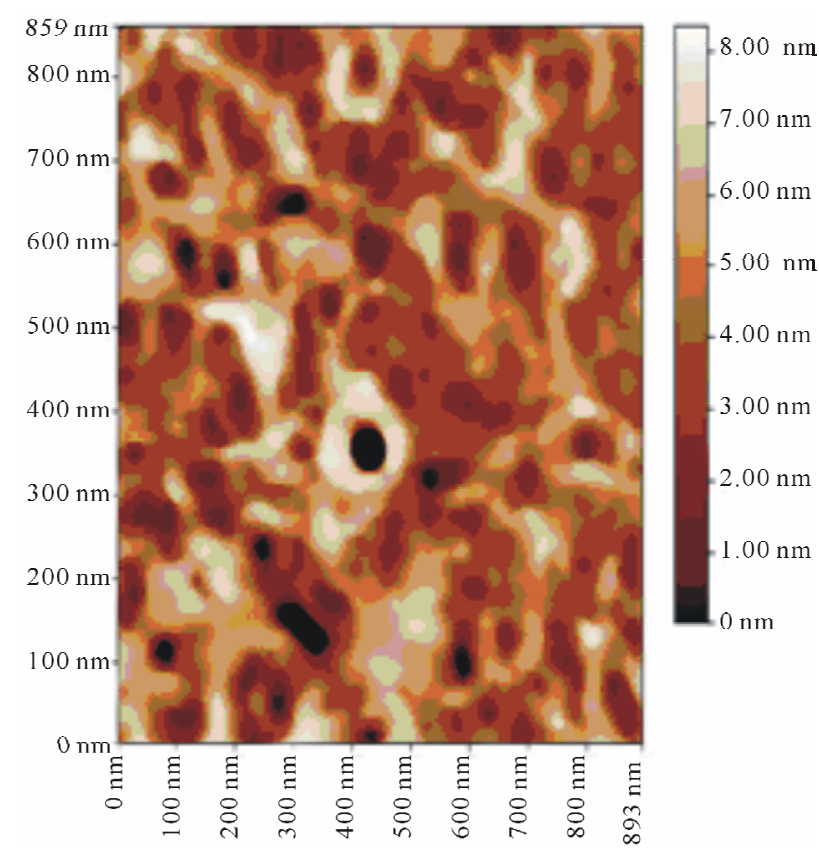

(a)

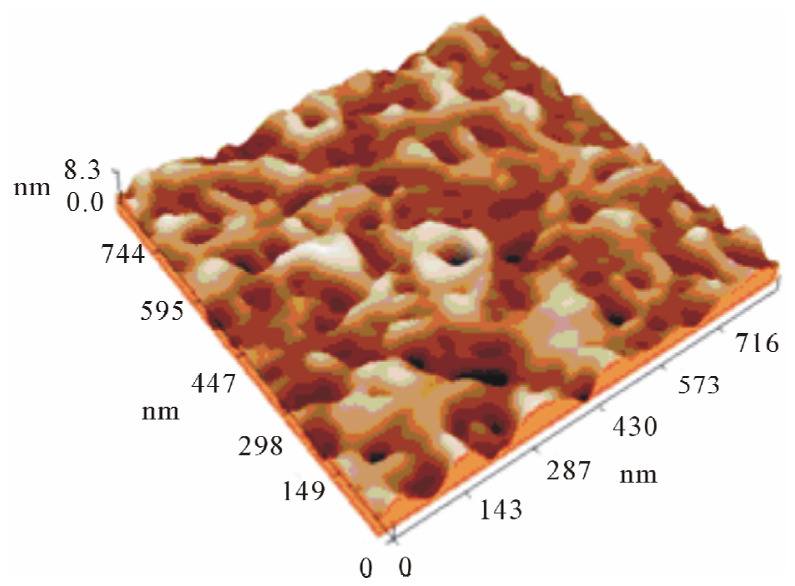

(b)

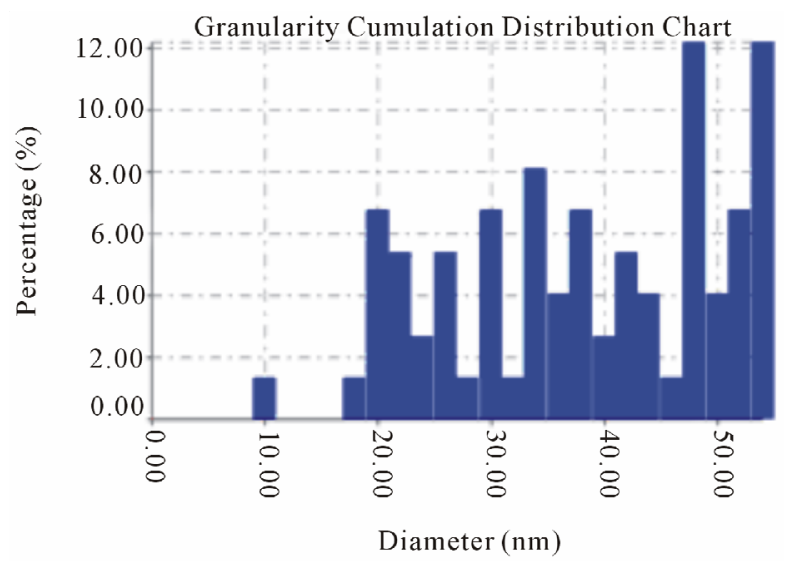

(c)

Figure 3. Atomic force microscope topographic views of AAO ionized during 3 hour (a) 2D image (b) 3D and (c) normal granulites distribution report of AAO. 
mina on the way to the conventional hexagonal pattern.

However, the results showed the average pore diameter linearly increased with anodization time. This increase in the pore diameter can be related as a result of prolonged etching of alumina in the acidic electrolyte used for anodizing. In addition, the complex network of pores containing subpores disappears due to the electrolyte etching action. This confirms that the pore diameter can be controlled by the anodization time. This is in agreement with the results obtained by Zaraska et al. and Li et al. $[11,13]$.

It was noticed that by increasing the duration of anodization led to the increase of the films roughness due to the increasing of thickness these results are similar to those reported by Wei et al. [15] who argue that the thickness of the porous oxide film was affected by the anodizing time. These results can be explained through the film morphology, as the film thickness increases the grain size increases too. The surface roughness increases with increasing grain size and appearance of enlarge pores caused by the extension of the aluminum surface, which led to possess an irregular structure and got separated by a thick pore walls. Similar results have been observed by Alam et al. [6].

AFM results for the AAO nanopurs films prepared at a different duration of anodization are summarized in Table 1.

It is worth noting that with a significant extension of the anodizing time a considerable improvement of pore arrangement have not been observed. Therefore, further study is needed to find how the surface condition of aluminum foil can modify homogeneity of pores and significant improvement in surface roughness on an anodic alumina surface.

The mechanism of the formation $\mathrm{Al}_{2} \mathrm{O}_{3}$ film and nanopurs arrays is proposed by many authors [11,16-25] they assumed that nanotubes formed as a result of a competition between:

1) Field-assisted anodic oxidation, defined as the for-

Table 1. Atomic force micproscope results.

\begin{tabular}{cccc}
\hline \multirow{2}{*}{ AFM results } & \multicolumn{3}{c}{ Duration of anodization (hour) } \\
\cline { 2 - 4 } & 1 & 2 & 3 \\
\hline Pore size (nm) & 16.04 & 26.19 & 37.39 \\
Ra (Roughness Average) nm & 0.692 & 1.01 & 1.32 \\
Rq (Root Mean Square) nm & 0.835 & 1.17 & 1.61 \\
Ry (Peak-Peak) nm & 3.8 & 3.78 & 8.26 \\
Rz (Ten Point Height) nm & 3.8 & 3.77 & 7.55 \\
Rk (Core Roughness Depth) nm & 2.43 & 3.39 & 4.39 \\
Rvk (Reduced Valley Depth) nm & 1.25 & 0.0073 & 1.46 \\
\hline
\end{tabular}

mation of the layer under applied electric field and Chemical filed/assisted dissolution of aluminum oxide (or alumina) at the oxide/electrolyte interface. The dissolution and thinning of the oxide layer is mainly due to the hydration reaction of the formed oxide layer.

2) Alumina formation at the oxide/aluminum interface. The oxidation takes place at the entire metal/oxide interface mainly by the migration of oxygen containing ions $\left(\mathrm{O}_{2}{ }^{-}\right.$or $\left.\mathrm{OH}^{-}\right)$from the electrolyte.

Pores are first formed at certain micro-rough region where the current density is concentrated on after the formation of a steady barrier layer and then the pores grow perpendicularly to the surface with equilibrium of field-enhanced oxide dissolution at the oxide electrolyte interface and oxide growth at the metal/oxide interface. The formed alumina is assumed to be $\mathrm{Al}_{2} \mathrm{O}_{3}$. Therefore, the atomic density of aluminium in alumina is by a factor of 2 lower than in metallic aluminium. This means that the volume of the anodized alumina expands to about twice the original volume. This volume expansion leads to compressive stress during the oxide formation in the oxide/metal interface. The expansion in the vertical direction pushes the pore walls upwards. Meanwhile, the repulsive interaction between the pores drives the structural adjustment to form pore arrays like in a honeycomb. The barrier layer at the bottom of the nanotubes (at the metal/oxide interface) acts as resistance to the flow of ions, which needs to move through the anodic film to keep the oxidation active. Greater resistance can be overcome by increasing the applied anodization voltage, which can cause the increase of the longitudinal growth of the pores due to the lager migration velocity of the reactive cation and anion ions, which can be confirmed by the thickening of the AAO the horizontal growth also leads to the enlargement of the pore diameter and spacing. Meanwhile, the adjusted velocity among the pores cannot keep up with the growth in velocity of the AAO and the features of the order of the structure might decrease. Moreover the degree of the volume expansion of aluminum determines either the formation of self-ordered pores or the formation of disordered pores.

\section{Conclusion}

Anodic aluminium oxide (AAO) film with the nanoporous was successfully prepared by the use of a two-steps anodized method. This study confirms that the duration of anodization is a conditional parameter that strongly influences the porous diameters and the roughness of the films. It was obtained that the pore size increased $(16,26$ and $37 \mathrm{~nm})$ in (1, 2 and 3 hour) respectively.

\section{Acknowledgements}

I want to thank Dr. Kareem AL-Samuraee for his sup- 
porting to carry out this work.

\section{REFERENCES}

[1] S. Shingubara, "Fabrication of Nanomaterials Using Porous Alumina Templates," Journal of Nanoparticle Research, Vol. 5, No. 1, 2003, pp. 17-30. doi:10.1023/A:1024479827507

[2] N. Taşaltina, S. Öztürka, H. Yüzerb and Z. Z. Öztürk, "Simple Fabrication of Highly Ordered AAO Nanotubes," Journal of Optoelectronic and Biomedical Materials, Vol. 1, No. 1, 2009, pp. 79-84.

[3] S. Phok, S. Rajaputra and V. P. Singh, "Copper Indium Diselenide Nanowire Arrays by Electrodeposition in Porous Alumina Templates," Nanotechnology, Vol. 18, No. 47, 2007, pp. 1-8.

[4] V. Sadasivan, C. P. Richter, L. Menon and P. F. Williams, "Electrochemical Self-Assembly of Porous Alumina Templates," 2005.

[5] H. Masuda and K. Fukuda, "Ordered Metal Nanohole Arrays Made by a Two-Step Replication of Honeycomb Structures of Anodic Alumina," Science, Vol. 268, No. 5216, 1995, pp. 1466-1468. doi:10.1126/science.268.5216.1466

[6] C. Sousab, D. C. Leitaoa, J. Venturaa, A. M. Pereiraa, M. Amadoa, J. B. Sousaa and J. P. Araújo, "Nanoporous SelfOrganized Anodic Alumina Templates,” 2007.

[7] P. Chowdhury, K. Raghuvaran, M. Krishnan, H. C. Barshilia and K. S. Rajam, "Effect of Process Parameters on Growth Rate and Diameter of Nanoporous Alumina Templates," Bulletin of Materials Science, Vol. 34, No. 3, 2011, pp. 423-427. doi:10.1007/s12034-011-0104-6

[8] K .M. Alam, A. P. Singh, S. C. Bodepudi and S. Pramanik, "Fabrication of Hexagonally Ordered Nanopores in Anodic Alumina: An Alternative Pretreatment," Surface Science, Vol. 605, No. 3-4, 2011, pp. 441-449. doi:10.1016/j.susc.2010.11.015

[9] X. Wang and G.-R. Han, "Fabrication and Characterization of Anodic Aluminum Oxide Template." Microelectronic Engineering, Vol. 66, No. 1-4, 2003, pp. 166-170. doi:10.1016/S0167-9317(03)00042-X

[10] K. Nielsch, J. Choi, K. Schwirn, R. B. Wehrspohn and U. Go1sele, "Self-Ordering Regimes of Porous Alumina: The 10\% Porosity Rule," Nano Letters, Vol. 2, No. 7, 2002, pp. 677-680.

[11] F. Li, Z. Zang and R. M. Metzger, "On the Growth of Highly Ordered Pores in Anodic Aluminum Oxide," Chemistry of Materials, Vol. 10, No. 9, 1998, pp. 2470-2480. doi:10.1021/cm980163a

[12] H. J. Oh, J. H. Lee, Y. Jeong, S. J. Suh and C. S. Chi, "A Control of the Nanopore Regularity in Alumina Layer," Materials Science Forum, Vol. 475-479, 2005, pp. 39193922. doi:10.4028/www.scientific.net/MSF.475-479.3919
[13] L. Zaraska, G. D. Sulka1 and M. Jaskuła, "Properties of Nanostructures Obtained by Anodization of Aluminum in Phosphoric Acid at Moderate Potentials," Journal of Physics: Conference Series, Vol. 146, No. 1, 2009, Article ID: 012020.

[14] W. Lee, K. Nielsch and U. Gosele, "Self-Ordering Behavior of Nanoporous Anodic Aluminum Oxide (AAO) in Malonic Acid Anodization," Nanotechnology, Vol. 18, No. 47, 2007, Article ID: 475713.

[15] W. Wei, S. Li, J. Liu and J. Zhang, "Size-Controlled Synthesis of Highly Ordered Nanoporous AAO Membranes," Nanoscience, Vol. 11, 2006, pp. 157-160.

[16] Y. Xu, G. E. Thompson and G. C. Wood, "Mechanism of Anodic Film Growth on Aluminium," Transactions of the Institute of Metal Finishing, Vol. 63, 1986, pp. 98-103.

[17] O. Sullivan and G. C. Wood, "The Morphology and the Mechanism of Formation of Porous Anodic Films on Aluminium," Proceedings of the Royal Society A, Vol. 317, 1970, pp. 511-543.

[18] V. P. Parkhutik and V. I. Shershulsky, "Theoretical Modeling of Porous Oxide Growth on Aluminum," Journal of Physics D: Applied Physics, Vol. 25, No. 8, 1992, pp. 1258-1263. doi:10.1088/0022-3727/25/8/017

[19] J. Siejka and C. Ortega, "Study of Field-Assisted Pore Formation in Compact Oxide Films on Aluminium," Journal of the Electrochemical Society, Vol. 124, No. 6, 1977, pp. 883-891. doi:10.1149/1.2133446

[20] G. E. Thompson and G. C. Wood, "Anodic Films on Aluminum," In: J. C. Scully, Ed., Treatise on Material Science and Technology, Academic Press, New York, 1983.

[21] K. Shimizu, K. Kobayashi, G. E. Thompson and G. C. Wood, "A Novel Marker for the Determination of Transport Numbers during Anodic Oxide Barrier Growth of Aluminum," Philosophical Magazine Part B, Vol. 64, No. 3, 1991, pp. 345-353. doi:10.1080/13642819108207625

[22] O. Jessensky, F. Múller and U. Gósele, "Self-Organized Formation of Hexagonal Pore Arrays in Anodic Alumina," Applied Physics Letters, Vol. 72, No. 10, 1998, pp. 1173-1175. doi:10.1063/1.121004

[23] A. P. Li, F. Müller, A. Birner, K. Nielsch and U. Gösele, "Hexagonal Pore Arrays with a 50 - 420 nm Interpore Distance Formed by Self-Organization in Anodic Alumina," Journal of Applied Physics, Vol. 84, No. 11, 1998, pp. 6023-6027. doi:10.1063/1.368911

[24] A. P. Li, F. Müller, A. Birner, K. Nielsch, U. Gösele and J. Vac, "Polycrystalline Nanopore Arrays with Hexagonal Ordering on Aluminum," Journal of Vacuum Science \& Technology A, Vol. 17, No. 4, 1999, pp. 1428-1432.

[25] G. E. Thompson, R. C. Furneaux, G. C. Wood, J. A. Richardson and J. S. Goode, "Nucleation and Growth of Porous Anodic Films on Aluminum," Nature, Vol. 272, No. 5652, 1978, pp. 433-435. doi:10.1038/272433a0 